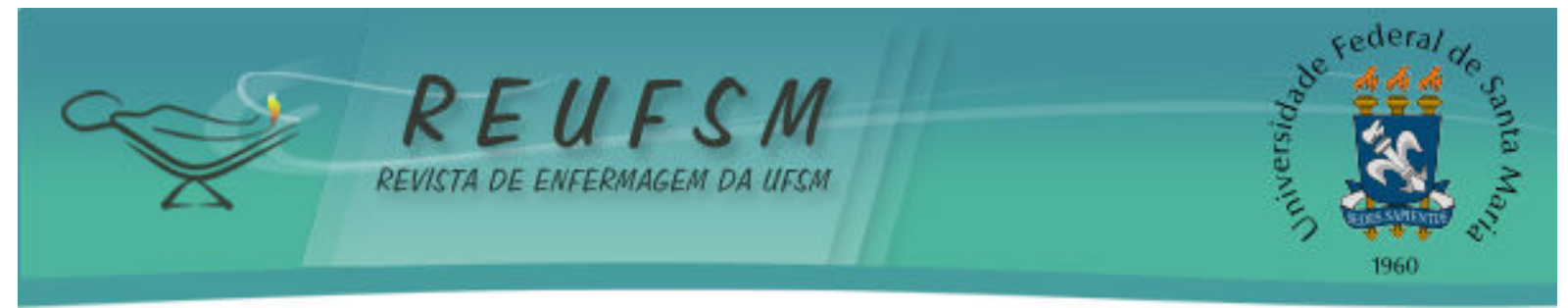

ARTIGO ORIGINAL

\title{
COMPETÊNCIAS DO PROFISSIONAL DA SAÚDE PARA O CUIDADO DA PESSOA COM AMPUTAÇÃO
}

\author{
HEALTH PROFESSIONAL'S COMPETENCES FOR THE CARE OF THE PERSON WITH \\ AMPUTATION
}

\section{COMPETENCIAS DEL PROFESIONAL DE LA SALUD PARA LA ATENCIÓN A LA PERSONA CON AMPUTACIÓN}

Doi: $10.5902 / 2179769218226$

Mara Ambrosina de Oliveira Vargas ${ }^{1}$

Dulcinéia Ghizoni Schneider ${ }^{2}$

Erika Yuriko Kinoshita ${ }^{3}$

Micheli Leal Ferreira ${ }^{4}$ Soraia Dornelles Schoeller ${ }^{5}$ Flávia Regina Souza Ramos ${ }^{6}$

RESUMO: Objetivo: analisar de que modo a competência do profissional da saúde se expressa no cuidado à pessoa com amputação. Método: estudo exploratório descritivo, desenvolvido em cinco hospitais públicos da grande Florianópolis. Realizado entrevista semiestruturada com 19 profissionais da saúde no período de dezembro de 2012 a março de 2013. Os resultados foram analisados na perspectiva da análise de conteúdo. Resultados: predomina a competência técnica, oriunda de uma formação que prioriza o conhecimento técnico-científico. A competência organizacional ou metódica nas ações dos profissionais da saúde é evidenciada pela capacidade de auto planejamento e autoorganização com flexibilidade e criatividade nas ações. A competência comunicativa e a competência sociopolítica precisam maior desenvolvimento. Conclusão: apesar dos participantes manifestarem consciência das implicações éticas e do compromisso social do seu trabalho, não há autonomia de ação.

Descritores: Competência Profissional; Amputação; Equipe de Assistência ao Paciente; Atenção à saúde.

Abstract: Aim: to analyze how the health professionals' competence is expressed on the care for the amputee patient. Method: exploratory and descriptive study, conducted in five public hospitals in Florianópolis. Semi-structured interviews were conducted among 19 health professionals from December 2012 to March 2013. The results were analyzed

\footnotetext{
${ }^{1}$ Professora Adjunta da Graduação e Pós Graduação do Departamento de Enfermagem da Universidade Federal de Santa Catarina, Doutora em Enfermagem pela Universidade Federal de Santa Catarina, Florianópolis/SC Brasil, e-mail: mara@ccs.ufsc.br;

2 Professora Adjunta do Departamento de Enfermagem da Universidade Federal de Santa Catarina, Doutora em Enfermagem pela Universidade Federal de Santa Catarina, Florianópolis/SC - Brasil, e-mail: dulcineiags@gmail.com;

3 Enfermeira Graduada pela Universidade Federal de Santa Catarina, Florianópolis/SC - Brasil, e-mail: erikayk167@gmail.com;

4 Doutoranda do Programa de Pós Graduação em Enfermagem da Universidade Federal de Santa Catarina, Mestre em Enfermagem pelo Programa de Pós Graduação em Enfermagem da Universidade Federal de Santa Catarina, Florianópolis/SC - Brasil, e-mail: micheli_leal@yahoo.com.br;

${ }^{5}$ Professora Adjunta do Departamento de Enfermagem da Universidade Federal de Santa Catarina, Doutora em Enfermagem pela Universidade Federal de Santa Catarina, Florianópolis/SC - Brasil, e-mail: soraia@ccs.ufsc.br; ${ }^{6}$ Professora Associada da Graduação e Pós Graduação do Departamento de Enfermagem da Universidade Federal de Santa Catarina, Pós Doutorado em Educação pela Universidade de Lisboa, Florianópolis/SC - Brasil, e-mail: flavia.ramos@ufsc.br.
} 


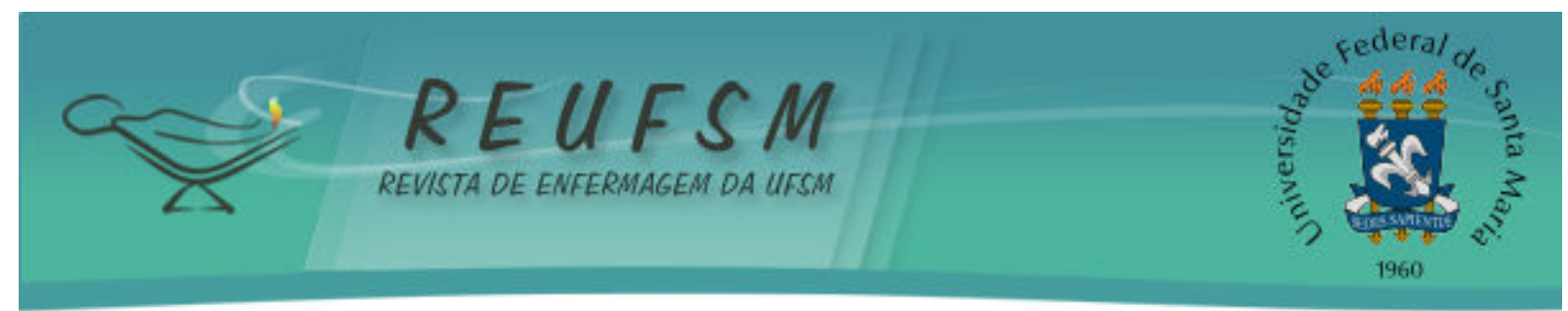

according to content analysis. Results: The technical competence was predominant, since it comes from a training that prioritizes technical and scientific knowledge. The organizational or methodical competence in the health professionals' action is evidenced by the ability of self-organization and self planning with flexibility and creativity in action. Communicative and socio-political competences need to be developed. Conclusion: although participants expressed awareness of the ethical and social implications of their work commitment, there is no autonomy of action.

Descriptors: Professional Competence; Amputation; Patient Care Team; Health Care.

Resumen: Objetivo: analizar cómo el profesional de salud se expresa en la atención a la persona con amputación. Método: estudio descriptivo y exploratorio, realizado en cinco hospitales públicos de Florianópolis. Fueron realizadas entrevistas semiestructuradas con 19 profesionales de salud desde diciembre 2012 hasta marzo 2013. Los resultados fueron analizados según el análisis de contenido. Resultados: predomina la competencia técnica, oriunda de una formación que prioriza el conocimiento técnico-científico. La competencia organizacional o metódica en la acciones de los profesionales de la salud es evidenciada por la capacidad de autoplaneamiento e auto-organización con flexibilidad y creatividad en las acciones. La competencia comunicativa y la competencia sociopolítica necesitan ser desarrolladas. Conclusiones: a pesar de los participantes manifestar consciencia de las implicaciones éticas y del compromiso social de su trabajo, no existe autonomía en su acción.

Descriptores: Competencia Profesional; Amputación; Grupo de Atención al Paciente; Atención a la Salud.

\section{INTRODUÇÃO}

O conceito de amputação está relacionado com a extração de parte do corpo com a intenção de proporcionar alívio nos sintomas, melhora/preservação das funções e qualidade de vida da pessoa que necessita da amputação. ${ }^{1}$ Os acidentes de trânsito são responsáveis por cerca de $56 \%$ das causas externas de amputação. ${ }^{2}$ Dentre as amputações eletivas, evidencia-se uma relação direta entre a idade avançada e as doenças crônico degenerativas. $\mathrm{E}$, os motivos de amputação mais evidenciados relacionam-se às doenças vasculares, gangrenas, além das complicações diabéticas em menores proporções. ${ }^{1,3-5}$

As pessoas com amputação carecem adaptar-se à sua nova condição de vida, este processo possui um contexto amplo que requer intervenções necessárias para que sejam superadas as dificuldades/desvantagens impostas por ela, as quais são múltiplas, sistêmicas e variam de acordo com a nova condição de vida. Estima-se que $15,6 \%$ da população mundial, com 15 anos ou mais, possui algum tipo de deficiência e destes 2,2\% possuem dificuldade funcional. ${ }^{6}$

Conforme o censo realizado no Brasil em 2010 pelo Instituto Brasileiro de Geografia e Estatística (IBGE), aproximadamente $24 \%$ da população brasileira possui alguma deficiência e, desse número, cerca de $7 \%$ dessa população possuem deficiência motora. ${ }^{7}$ Desta forma a problemática da amputação possui repercussão social, e para realizar o cuidado e a reabilitação da pessoa amputada é necessário o trabalho multiprofissional. Para isso o profissional da área da saúde precisa desenvolver competências específicas acerca desse tema.

A competência é o assumir responsabilidade; é uma atitude social, antes de se constituir como um conjunto de conhecimentos profissionais. Entretanto, na maioria das situações, o assumir responsabilidade frente às situações de trabalho complexas, necessita de aquisição de novos conhecimentos. Desta forma, o profissional que aceita e se dispõe a 


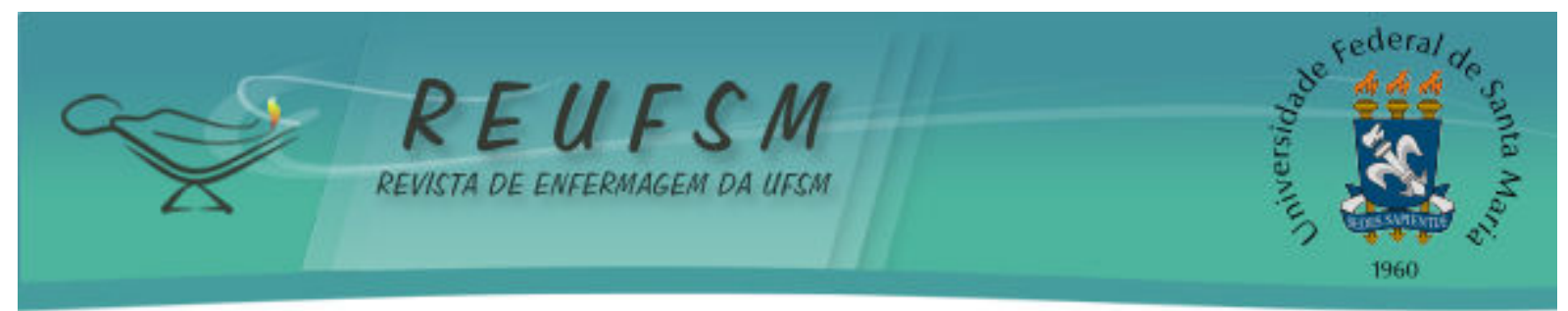

mobilizar-se, terá mais facilidade em desenvolver habilidades que uma pessoa que se mantem em posição defensiva ou de rejeição. ${ }^{8}$

De acordo com o parecer do Conselho Nacional de Educação (CNE) n $n^{\circ} 16 / 99$ e a resolução $n^{\circ}$ 04/99, que explicita as Diretrizes Curriculares Nacionais para a Educação Profissional de Nível Técnico e as competências profissionais para a Área de Saúde, a competência para o cuidar em saúde e enfermagem é:

\begin{abstract}
"Capacidade de mobilizar e articular conhecimentos, habilidades, atitudes e valores requeridos pelas situações de trabalho (imprevistas ou não) assumindo a responsabilidade do cuidado a partir da concepção de saúde como qualidade de vida, interagindo com equipe de saúde e com os usuários, percebendo suas necessidades e escolhas, valorizando sua autonomia para assumir sua própria saúde". 9:8
\end{abstract}

Neste contexto, as políticas públicas são importantes aliadas para que os profissionais de saúde desenvolvam as competências, de modo a possibilitar ações e intervenções necessárias às pessoas amputadas. A própria Constituição de 1988, em seu artigo 23, capítulo II, afirma que "é competência comum da União, Estados, Distrito Federal e Municípios, cuidar da saúde e assistência públicas, da proteção e garantia das pessoas portadoras de deficiências". ${ }^{10: 3}$

Há decretos e leis de apoio às pessoas com deficiência e sua integração social, prioridades ao atendimento, critérios de promoção da acessibilidade. Em 2002, através da Portaria $n^{\circ} 1.060$ de 5 de junho de 2002 foi instituída a Política Nacional de Saúde da Pessoa com Deficiência, resultado de "múltiplos movimentos e longa mobilização, nacional $e$ internacional, de muitos atores sociais e institucionais". ${ }^{10: 5}$

A competência e a prestação dos serviços do profissional têm como enfoque primordial a função de acautelamento dos riscos e resguardo do direito à vida, com qualidade. ${ }^{9}$ O profissional de saúde deve ser reflexivo, buscando o cuidado ao cliente de forma a preservar, respeitar e reconhecer a individualidade de cada pessoa. Entende-se que os profissionais competentes e engajados, podem contribuir no direito das pessoas amputadas viverem com dignidade. ${ }^{11}$

Nesta perspectiva, identificou-se que não há estudos que discutam de modo explícito a necessária competência do profissional da saúde para o cuidado da pessoa com amputação. De modo geral, predominam as temáticas que abordam a prevenção, as causas, as indicações, o momento do procedimento cirúrgico da amputação e os aspectos pós-amputação.

Mediante esta realidade, indaga-se: de que modo a competência do profissional da saúde se expressa no cuidado à pessoa com amputação nos hospitais públicos da grande Florianópolis. Logo, constitui-se como objetivo analisar de que modo a competência do profissional da saúde se expressa no cuidado à pessoa com amputação.

\title{
MÉTODO
}

Estudo exploratório descritivo que está inserido em um macro projeto denominado: "Pessoas submetidas à amputação clínica ou traumática: uma análise sob a perspectiva da bioética", apoiado pelo Conselho Nacional de Desenvolvimento Científico e Tecnológico, desenvolvido no nível de atenção da alta complexidade e compreendeu cinco hospitais públicos da grande Florianópolis com serviços especializados em traumatologia e ortopedia, fisiatria, cirurgia geral, cirurgia vascular e o Centro de Reabilitação. 


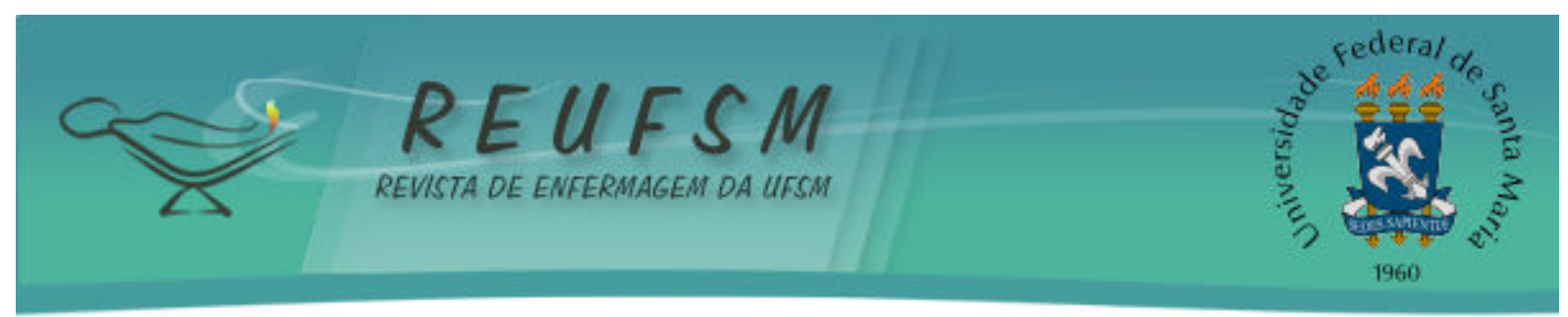

Os participantes do estudo foram profissionais da saúde envolvidos diretamente no cuidado à pessoa com amputação durante a hospitalização e a reabilitação. Mediante assinatura do Termo de Consentimento Livre e Esclarecido (TCLE), foram realizadas entrevistas semiestruturadas com 19 profissionais das diversas áreas de conhecimento, tais como médicos, enfermeiros, assistentes sociais, nutricionistas, psicólogos e fisioterapeutas, em seus locais de trabalho. A coleta de dados se deu no período de dezembro de 2012 a março de 2013. Definiu-se, previamente, que seriam entrevistados de três à seis profissionais de cada instituição, justamente, porque seriam os profissionais representantes de cada categoria profissional atuantes na unidade de internação que concentra as internações das pessoas submetidas à amputação. Assim, a escolha dos participantes foi por conveniência, de modo a abranger diferentes categorias profissionais e etapas da assistência, sendo excluídos os profissionais que estavam afastados por férias ou licença de saúde.

Em duas instituições entrevistaram-se três profissionais, de cada uma, pelo motivo de não aceitação dos demais profissionais; em outras duas instituições, cinco profissionais, respectivamente, e em uma das instituições a equipe que atuava diretamente era composta por três categorias profissionais (médico, enfermeiro e assistente social).

Para conduzir as entrevistas foram elaboradas 10 questões para que os participantes respondessem, buscavam indagar acerca dos modos de desenvolver o cuidado à pessoa com amputação, da existência ou não de protocolo, das dificuldades no exercício profissional, do envolvimento com a reabilitação e a orientação da pessoa com amputação.

As entrevistas, com duração média de 60 minutos, foram gravadas em áudio e posteriormente transcritas e importadas para o software ATLAS Ti que auxiliou no processo de categorização dos dados. Categorizaram-se as falas dos participantes de acordo com as dimensões propostas pelo Parecer CNE no $16 / 99$ e a Resolução no 04/99, que explicita as Diretrizes Curriculares Nacionais para a Educação Profissional de Nível Técnico e as competências profissionais para a Área de Saúde, sendo uma proposta de educação profissional baseada em competências que devem tomar como base a competência humana para o cuidar em saúde-enfermagem. ${ }^{12} \mathrm{~A}$ análise dos dados ocorreu por Análise de Conteúdo. ${ }^{13}$

Os participantes foram identificados na sequência numeral, sexo e com a primeira letra da categoria profissional (exemplo $1,2,3$, seguido do sexo feminino e masculino = F; $M$ e seguido da profissão; exemplo: $1 \mathrm{~F} \mathrm{M}=$ participante 1 - feminino - médico). Para tal, adotaram-se as letras A, E, F, M, N, P para identificar, respectivamente, os profissionais: assistente social, enfermeiro, fisioterapeuta, médico, nutricionista e psicólogo. 0 estudo foi submetido ao Comitê de Ética em Pesquisa (CEP) pela Plataforma Brasil, sendo aprovado pelo CEP da Universidade Federal de Santa Catarina, número de parecer 95.521/2012. O estudo respeitou os preceitos éticos de pesquisa com seres humanos.

\section{RESULTADOS}

A competência técnica inclui o conhecimento peculiar de cada profissão da saúde, envolvida no processo de cuidar, a articulação de informações necessárias na rede de relações, o uso de equipamentos e a compreensão de todo o processo. ${ }^{12}$ Relaciona-se às especificidades de cada profissão que, em conjunto com outros profissionais e com as redes, integra o cuidado com o objetivo de satisfazer as necessidades da pessoa com amputação.

As falas dos profissionais, a seguir, demonstram a articulação da competência técnica no cuidado à pessoa com amputação: 


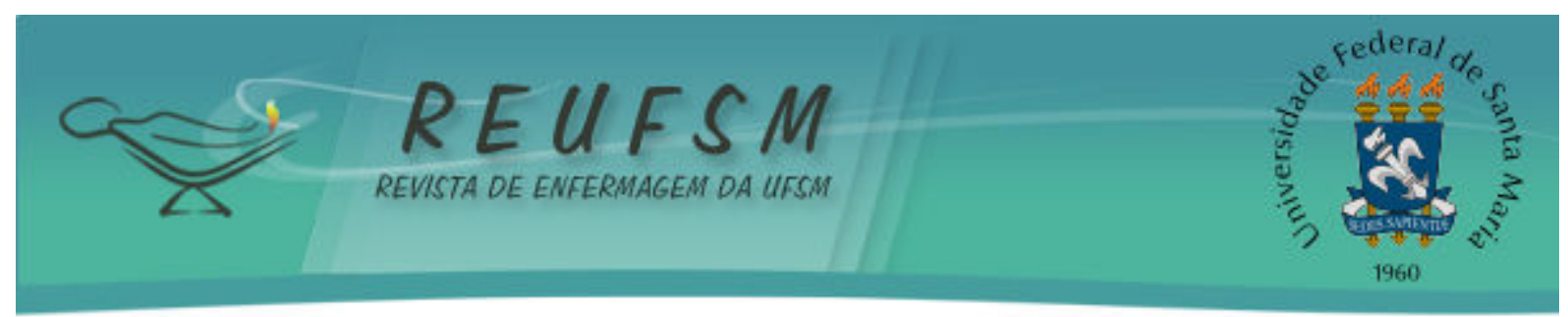

na consulta de enfermagem detecta-se os fatores estressores, necessários de serem trabalhados. 0 mais comum são as comorbidades relacionadas com a amputação, o trabalho de orientação nessa área primária.(1FE)

estes pacientes chegam meses depois da amputação, tem que dessensibilizar esse coto porque normalmente o paciente tem a dor fantasma. $O$ trabalho propioceptivo visa ensinar o paciente a fazer massagem, toque, passar superfícies diferentes na pele, banho de sol, o próprio enfaixamento, porque o coto tem que ficar num sentido de cone, pra que essa prótese tenha esse encaixe.(2FE)

a amputação feita pela cirurgia vascular é realizada em paciente com doença arterial periférica grave, ou com infecção de membro inferior ou superior grave. Explicamos a gravidade da doença, o risco de vida que a pessoa está correndo se não realizar a amputação e o familiar ou o paciente assinam um termo de consentimento da amputação. (14MM)

sou responsável pela avaliação clínica inicial do paciente que é amputado no Centro de Reabilitação. Faço consulta médica e uma avaliação da condição do paciente e do coto. (3MM)

treinamos o uso da prótese e ele [paciente] vai andando embora. [...] revisamos a prótese três vezes, a cada seis meses. Orientamos para não trocar o solado do calçado porque a prótese é alinhada com o calçado que ele traz, para não ter pressão onde não deve. [...] acompanhamos sempre o paciente. (5MF)

fazemos avaliação nutricional, entrevista, anamnese, no pré ou no pós-operatório para auxiliar a cicatrização do paciente. Há um suplemento para isso, que nossa instituição tem nas cotas. (17FN)

trabalho a autoestima, as possibilidades que a pessoa tem enquanto aguarda a prótese, algumas com a prótese podem retornar à

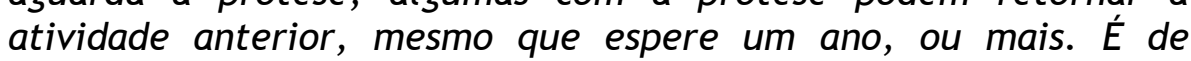
readaptação a alternativas de trabalho, de aplicação do tempo, de participação em grupos, para que esse período possa ser mais saudável emocionalmente. É difícil para eles esperar pela protetização, pela dificuldade do coto em si, tramites burocráticos ou de concessão de próteses. (7FP)

A competência comunicativa refere-se à expressão e comunicação com o grupo, visando à cooperação, o trabalho em equipe, o diálogo, a negociação. ${ }^{14-15}$ Nesta direção, ao cuidar da pessoa com amputação, os profissionais necessariamente precisam obter informações do paciente e família, e articular a integralidade do cuidado com toda a equipe de saúde. Suas falas contextualizam, de diferentes modos, esta competência:

pedimos para o assistente social orientar a parte de encaminhamento para a reabilitação. O serviço social tem a ficha 


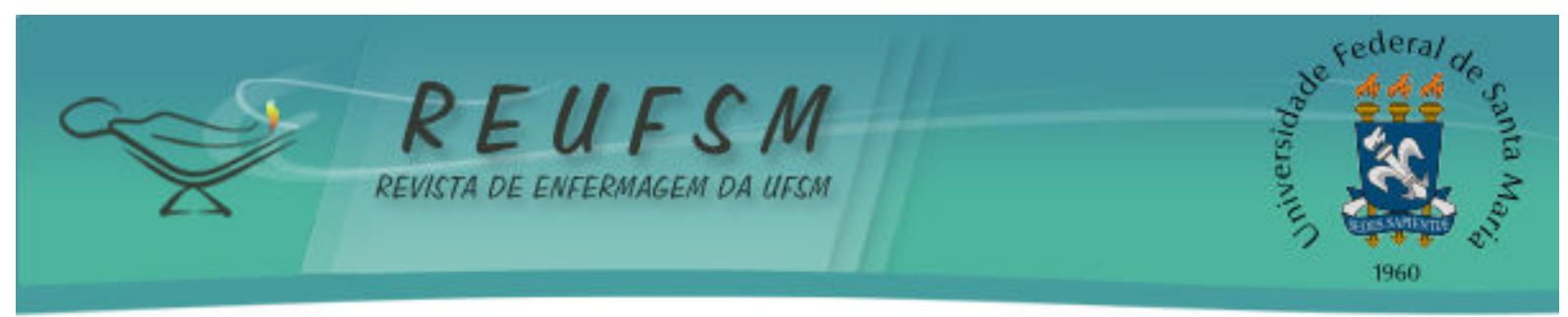

de solicitação de muletas, cadeira de rodas, todo o material de apoio para o paciente. Ele traz e solicitamos que o médico preencha e o assistente social orienta. (10ME)

nossa primeira preocupação é relacionar a amputação às novas possibilidades do paciente [...] informamos a família da situação, da oportunidade que pode se apresentar e fazemos esse vínculo com a reabilitação, normalmente aqui na Associação Catarinense de Reabilitação e no município do paciente. Conheci o esposo de uma paciente, cardíaca e ele era amputado há vinte anos e andava com duas muletas. $O$ sonho dele era comprar uma prótese [...] encaminhei ele para a reabilitação, para o médico ortopedista, o posto de saúde, quem pudesse encaminhá-lo para a reabilitação. Ele prometeu que quando estivesse com a prótese viria me ver, acho que ele já esqueceu de mim! Mas torço muito para que hoje ele tenha a prótese.(19FA)

eu acredito que na equipe todos falam uma linguagem parecida. $\dot{E}$ uma coisa padronizada e que é de conhecimento da equipe de enfermagem tanto pode acontecer, as orientações pelo enfermeiro e pelo técnico. (13FE)

Ao se considerar as competências organizacionais ou metódicas, que estão relacionadas à capacidade de auto planejar-se, de auto-organizar-se, de estabelecer métodos próprios, de desenvolver a flexibilidade no processo de trabalho, exercitando a criatividade, utilizando os seus conhecimentos nas diversas situações encontradas no mundo do trabalho. ${ }^{12}$ Evidencia-se, nas falas dos participantes a seguir, que o desenvolvimento desta competência é complexo:

não existe o psicólogo na Instituição, o enfermeiro se envolve com o cuidado, com a ferida e, também, com a família e com o lado emocional e psicológico do paciente.(15FE)

atendemos pessoas que já tem comprometimento, um renal crônico, amputado, câncer de próstata, não tem perspectiva de uma evolução positiva. [...] Mas você sabe que em determinados casos os processos vão ser mais longos ou que você não vai chegar muitas vezes àquele objetivo final que é a protetização. Você precisa de diversos profissionais, diversas áreas. (2FE)

Ao abordar as competências sociopolíticas, reflete-se acerca das implicações éticas do trabalho, a autonomia de ação e o compromisso social e de exercício da cidadania. ${ }^{12}$ Salienta-se que no cuidado à pessoa com amputação, deve-se conhecer a realidade em que ela vive; quais são as demandas necessárias ao cuidado, quais redes de apoio dispõem para então definir as melhores estratégias de cuidado. A seguir, observa-se nas falas os diferentes aspectos:

como assistente social procuro inicialmente entrevistar a pessoa para entender o contexto social e ao longo da internação vai ser decidido a necessidade ou não da amputação e como nossos 


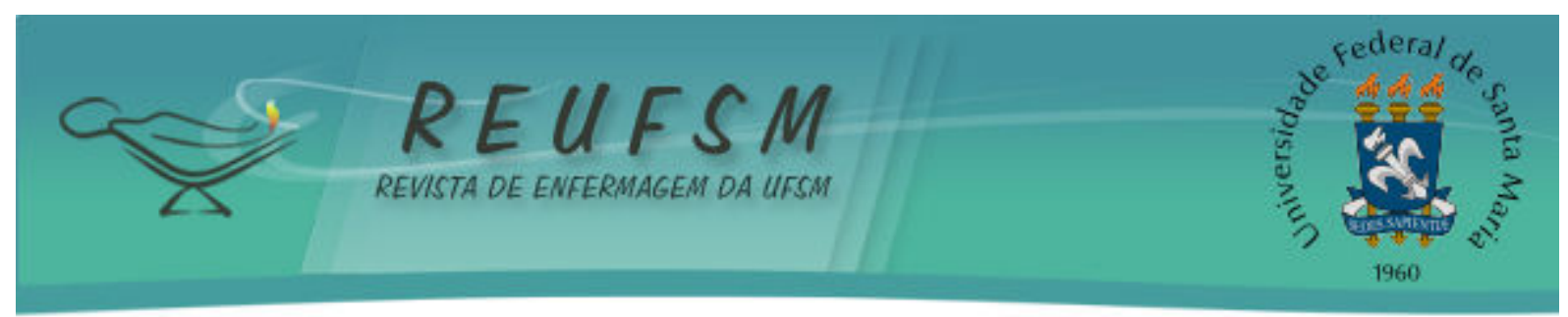

pacientes vem de longe, [...] procuro ver a forma como atuarei. Me organizo com o município pra fazer uma emenda boa entre o tratamento aqui e o serviço de referência lá no município dela.(19FA)

às vezes a lesão não vai totalmente cicatrizada e ele depende do posto. Nós temos que saber a realidade daquele posto, os recursos para cicatrizar a lesão e orientamos o que ele vai fazer para que cicatrize mais rápido, para usar prótese. $(8 \mathrm{FE})$

se ela é de outro município e tem que voltar para avaliação do coto em um mês, tento conciliar, até por questões de transporte. No caso da pessoa precisar muito e voltará em seis meses no Centro de Reabilitação, marco um retorno, geralmente um mês que é um tempo médio da pessoa conseguir entrar na lista, ser chamada no posto de saúde para ver como é que está a situação. Se tiver alguma intercorrência mais séria ou algum risco emocional, faço outra intervenção.(12FP)

\section{DISCUSSÃO}

Mediante os resultados apresentados, ressalta-se a magnitude da temática "competência profissional", entendendo que o desenvolvimento destas são cruciais para o sucesso e o empoderamento profissional nas ações e na assistência em saúde.

Destaca-se que uma equipe de saúde multiprofissional não é apenas um grupo de diferentes pessoas atuando juntas e desenvolvendo cada qual sua competência técnica específica. É, antes, um grupo interdependente e engajado que se articulam visando à obtenção dos melhores resultados. ${ }^{15}$

Dentre as quatro competências profissionais anteriormente elucidadas, a competência técnica é compreendida e referida pelos distintos profissionais de saúde que contribuíram com a pesquisa. Observa-se que uma parcela significativa das competências técnicas específicas são adquiridas durante o processo de formação. Mas, existem situações não vivenciadas previamente e que passam a constituir o cotidiano destes trabalhadores. Agrega-se a isto, a demanda por um contínuo desenvolvimento no domínio das políticas públicas e do atual sistema de saúde brasileiro para adequar o tratamento a cada indivíduo e suas necessidades específicas.

Um fato inegável é a transcendência do cuidado na execução de técnicas, em uma época de transformações dinâmicas, mas com demonstrações concretas das limitações na área da saúde, uma vez que, a patologia não justifica mais o doente. Emerge um espaço de construção de relações solidárias, amparadas no respeito às diferenças e visando o bem comum, onde o sujeito enfermo e o cuidador unem suas forças para a busca do bem-estar, da autonomia e da felicidade. ${ }^{16}$

Ao cuidador cabe o domínio de suas competências técnicas específicas para que em dada situação, conheça os tratamentos disponíveis, bem como, as possibilidades de autotratamento para assegurar que cada pessoa, seja ela com amputação ou não, recebam uma assistência individualizada e integral. ${ }^{17}$

$\mathrm{Na}$ competência comunicativa, os resultados da pesquisa demonstram a incipiência na comunicação entre profissionais de diferentes níveis de atenção, o que pode limitar o acesso da pessoa com amputação aos cuidados mais adequados à sua adaptação a esta condição de saúde. Neste sentido, o prontuário eletrônico nas Redes de Atenção à Saúde 


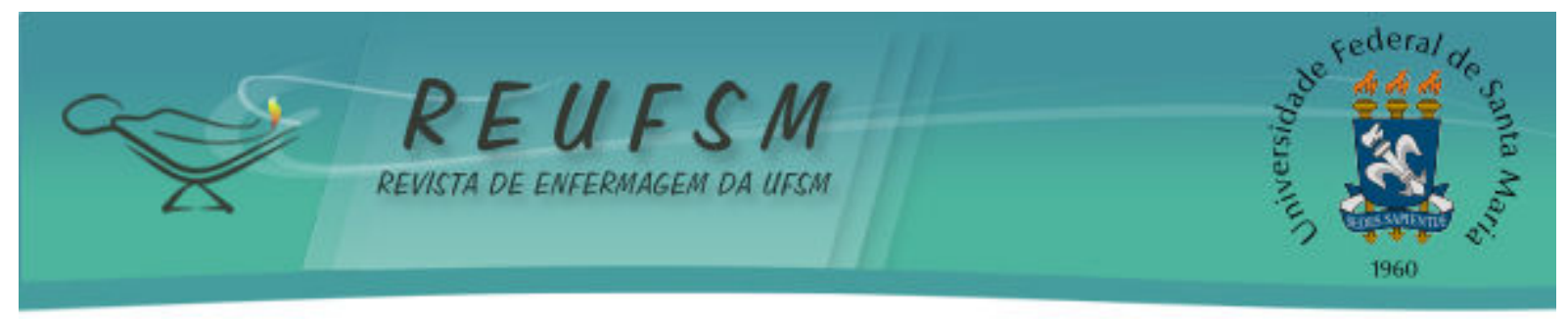

(RAS), é uma possibilidade de efetivar um sistema de referenciamento e contrarreferenciamento de pessoas e estão focados em promover a integração dos pontos de atenção à saúde com eficácia. ${ }^{8}$

É imprescindível, também, poder contar com profissionais com espírito de equipe e de cooperação entre seus integrantes. Isto demanda aos mesmos a necessidade de estabelecer e compartilhar uma visão comum e que estes aprendam a solucionar problemas pela sua competência comunicativa, através do diálogo e da negociação de modo a maximizar as habilidades singulares de cada um. ${ }^{15}$

Outro ponto crítico na competência comunicativa evidencia a necessidade dos profissionais obterem informações do paciente e família, e articular a integralidade do cuidado com a equipe multiprofissional, uma vez, que se trata de um processo contínuo que exige a participação e o envolvimento de toda a equipe, do paciente, da família e das redes de atenção à saúde. Mediante esta realidade, questiona-se: se uma pessoa espera há 20 anos por uma prótese, qual ou quais são a(s) lacuna(s) do processo de assistência à saúde às pessoas com amputação?

Ao refletir sobre possíveis estratégias de resolução, direciona-se a análise para a terceira competência que é a organizacional ou metódica. Todos os profissionais da saúde, envolvidos no processo de cuidar, precisam ter a capacidade de se autoplanejar e organizar, cada qual estabelecendo métodos próprios, desenvolvendo suas ações com criatividade e flexibilidade. Isso pode se exemplificar na fala de um dos enfermeiros participantes do estudo, ao manifestar o que faz mediante a inexistência do psicólogo no serviço.

O enfermeiro ao se deparar com uma limitação em seu ambiente de trabalho se organizou, e com sua flexibilidade e comprometimento com o cuidado, forneceu o apoio emocional que aquela pessoa e sua família demandaram. Todavia, é necessário que a presença do psicólogo seja assegurada em todos os níveis de atenção, onde a atuação desse profissional pode contribuir para a promoção da qualidade de vida das pessoas, especificamente, em sua dimensão psicossocial.

Outro aspecto que pode ser abordado a partir da competência organizacional é o momento adequado para uma intervenção. A reabilitação de uma pessoa com amputação é longa e deve iniciar antes do momento da amputação. Nesta direção, diversas ações de reabilitação podem ser iniciadas e a atuação da equipe de saúde neste período, abrange avaliação física detalhada, explanação sobre o prognóstico funcional, esclarecimentos sobre dor fantasma e discussões sobre as metas de reabilitação de curto, médio e longo prazo. ${ }^{1,18,19,20,21}$

Finalmente, quanto à competência sociopolítica dos profissionais de saúde, notouse que os mesmos estão engajados com o princípio de que o indivíduo com amputação insere-se em uma comunidade e possui uma realidade social. Assim, para desenvolver um plano de cuidado integral é imprescindível conhecer as necessidades e com quais redes de apoio as pessoas com amputação podem contar e estar disposto a reformular este planejamento sempre que necessário.

Contudo, detectou-se a preocupação dos profissionais quanto à questão do acesso a assistência necessária pós-alta hospitalar. A Portaria $n^{\circ} 963$ de 27 de maio de 2013 define a Atenção Domiciliar (AD) no âmbito do SUS. O Serviço de Atenção Domiciliar (SAD) é financiado pelo Ministério da Saúde com eventual complementação dos Estados, do Distrito Federal e dos Municípios. Com a SAD quando clinicamente indicado, será designada uma equipe multiprofissional de atenção primária para dar suporte e complementar as ações de saúde da atenção domiciliar. ${ }^{22}$

A prática e o tempo de experiência profissional são indispensáveis ao aperfeiçoamento do domínio da função. A união de conhecimentos, de saber-científico, 


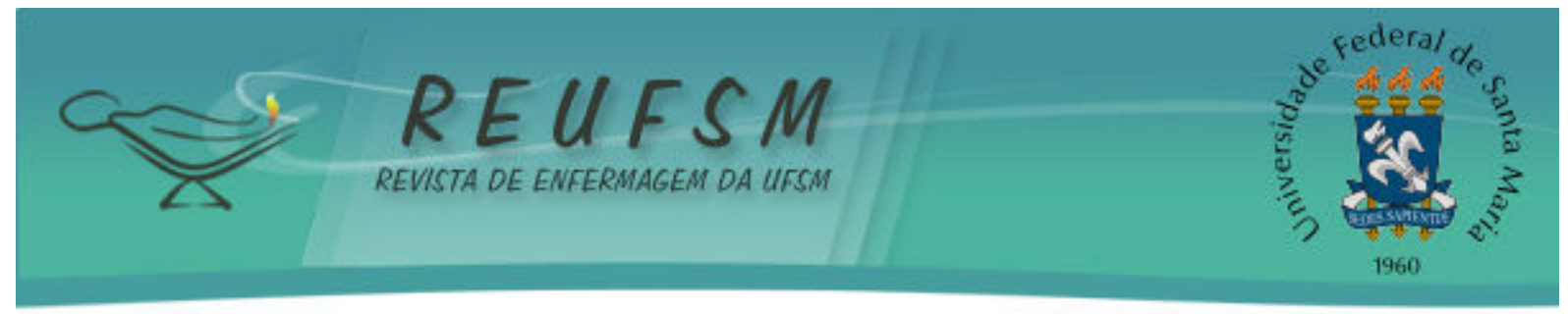

saber-ser, saber-prático e saber-informação, permitem o empoderamento e o aperfeiçoamento de competências na área da reabilitação. ${ }^{23}$

Falar em promover a autonomia e o autocuidado não é limitar tal ação ao simples fato da prescrição do profissional de saúde onde se aponta o que aquele indivíduo deve fazer. Deve-se reconhecer o papel central da pessoa em relação a sua saúde, aguçando o sentido de auto responsabilidade sanitária e fazendo com que o profissional de saúde transforme-se em um parceiro. ${ }^{18}$

\section{CONCLUSÃO}

Ao traçar um paralelo entre as ações desenvolvidas pelos profissionais da saúde que cuidam de pessoas com amputação e os quatro tipos de competências definidas pelo Ministério da Saúde, percebe-se que a competência técnica se sobressai em relação às demais já que o profissional em sua formação tem como maior referência o conhecimento técnico-científico.

A expressão da competência organizacional ou metódica nas ações dos profissionais da saúde no cuidado à pessoa com amputação apesar de complexa caracterizou-se como adequada, uma vez que os profissionais demonstraram capacidade de auto planejamento, auto-organização, flexibilidade e criatividade em suas ações.

A competência comunicativa mostrou-se ainda incipiente nas relações interprofissionais, com repercussões negativas no cuidado à pessoa com amputação. A falta de diálogo entre os profissionais limita a integralidade do cuidado e, de certa forma, demonstra falta de engajamento na resolução dos problemas de saúde de um indivíduo que necessita de um acompanhamento integral para atender suas necessidades e sua reabilitação e inserção social. E, a competência sociopolítica dos profissionais de saúde também precisa ser mais desenvolvida, pois apesar de muitos participantes terem consciência das implicações éticas e do compromisso social do seu trabalho, se faz necessário uma imersão por completa em cada caso, em cada realidade para que seja possível definir as melhores estratégias para o cuidado.

O presente estudo contribui para a área da saúde e enfermagem, na medida em que explora a complexidade de um trabalho que exige, efetivamente, a articulação de diferentes profissões para impactar na particularidade apresentada no cuidado prestado à pessoa com amputação, potencializando a sua recuperação e reabilitação. Logo, as instituições de saúde deveriam considerar a importância de manter uma equipe de saúde com os diferentes profissionais.

Considera-se uma limitação do presente estudo, justamente porque as respectivas instituições proporcionam uma atenção à pessoa, com amputação com uma equipe de profissionais "incompleta", a restrição do número de entrevistas aos diferentes profissionais da saúde.

\section{REFERÊNCIAS}

1. Marques AMFB, Vargas MAO, Schoeller SD, Kinoshita EY, Ramos FRS, Trombetta AP. 0 cuidado à saúde à pessoa com amputação: análise na perspectiva da bioética. Rev Texto Contexto Enferm [Internet]. 2014 [cited 2015 Jan 16]; 23(4). Avaliable from:<http: / /www.scielo.br/scielo.php?script=sci_arttext\&pid=S01

$0407072014000400898 \& \operatorname{lng}=p t \& n r m=i s o>$. 


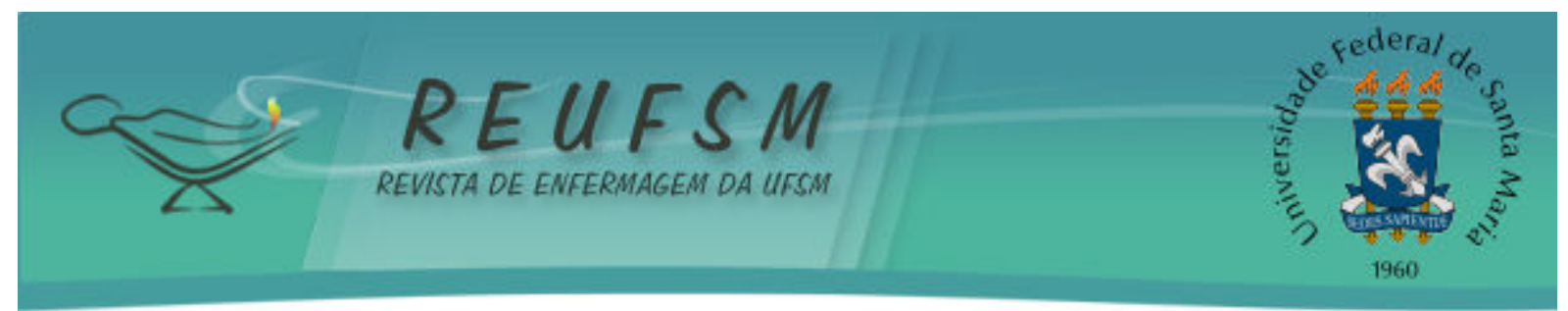

2. Omoke NI, Chukwu COO, Madubueze CC, Egwu AN. Traumatic extremity amputation in a Nigerian setting: patterns and challenges of care. IntOrthop [Internet]. 2012 [cited 2015 Jan 16]; 36(3): 613-8. Avaliable from: http://link.springer.com/article/10.1007\%2Fs00264011-1322-7/fulltext.html.

3. Henry AJ, Hevelone ND, Belkin M, Nguyen LL. Socioeconomic and Hospital-Related Predictors of Amputation for Critical Limb Ischemia. J Vasc Surg [Internet]. 2011 [cited 2015 Fev 10]; 53(2): 330-9. Avaliable from: http://www.sciencedirect.com/science/article/pii/S0741521410021634.

4. Alvarsson A, Sandgren B, Wendel C, Alvarsson M, Brismar K. A retrospective analysis of amputation rates in diabetic patients: can lower extremity amputations be further prevented? Cardio Vasc Diabetol [Internet]. 2012 [cited 2015 Jan 6]; 11(18):111. Avaliable from: http://www.ncbi.nlm.nih.gov/pmc/articles/PMC3362

773/pdf/1475-2840-11-18.pdf.

5. Horne MLA, Carolyn E. Complementary pain intervention pilot study in the acute hospitalization phase after lower extremity amputation surgery. The Scholar Ship [Internet]. $2014 \quad$ [cited $2015 \quad$ Mai 4$]. \quad$ Available from:http://thescholarship.ecu.edu/handle/10342/4422.

6. OMS. Organização Mundial da Saúde. Relatório mundial sobre a deficiência. São Paulo. 2012.

7. IBGE. Instituto Brasileiro de Geografia e Estatística. Censo 2010. 2010.

8. Fleury MTL, Fleury A. Construindo o Conceito de Competência. Rev Adm Contemp [Internet]. 2001 [cited 2015 Jan 05]; (5): 183-196. Available from: http: / / www.scielo.br/scielo.php?script=sci_arttext\&pid=S141565552001000500010.

9. Brasil. Referências conceituais para a organização do sistema de certificação de competências/Projeto de Profissionalização dos Trabalhadores na Área de Enfermagem Profae. Ministério da Saúde; Secretaria de Gestão de Investimentos em Saúde. Brasília, jul. 2000.

10. Brasil. Portaria $n^{\circ} 4.279$, de 30 de dezembro de 2010. Estabelece diretrizes para organização da Rede de Atenção à Saúde no âmbito do Sistema Único de Saúde. Diário Oficial da União. Poder Executivo. Brasília, 31 dez. 2010. Seção 1.

11. Schoeller SD, Silva DMGV, Vargas MAO, Borges AMF, Pires DEP, Bonetti A. Características das pessoas amputadas atendidas em um centro de reabilitação. Rev enferm UFPE [Internet]. 2013 [cited 2015 Jan 13]. 7(2):445-51. Avaliable from: http://www.revista.ufpe.br/revistaenfermagem/index.php/revista/article/download/335 $1 / 5326$.

12. Lovell A, Bailey J, Kingdon A, Gentile D. Working with people with learning disabilities in varying degrees of security: nurses' perceptions of competencies. J of Advanced Nursing. 2014;70(9): 2041-2050. DOI: 10.1111/jan.12362.

13. Minayo MCS. O desafio do conhecimento: pesquisa qualitativa em saúde. 16a ed. São Paulo (SP): Hucitec; 2014.

14. Marques CMS, Egry EY. As competências profissionais em saúde e as políticas ministeriais. Rev Esc Enferm USP. São Paulo. 2011; 45(1): 187-193.

15. Mendes EV. As redes de atenção à saúde. Organização Pan-Americana da Saúde. Brasília. 2011; 549. 


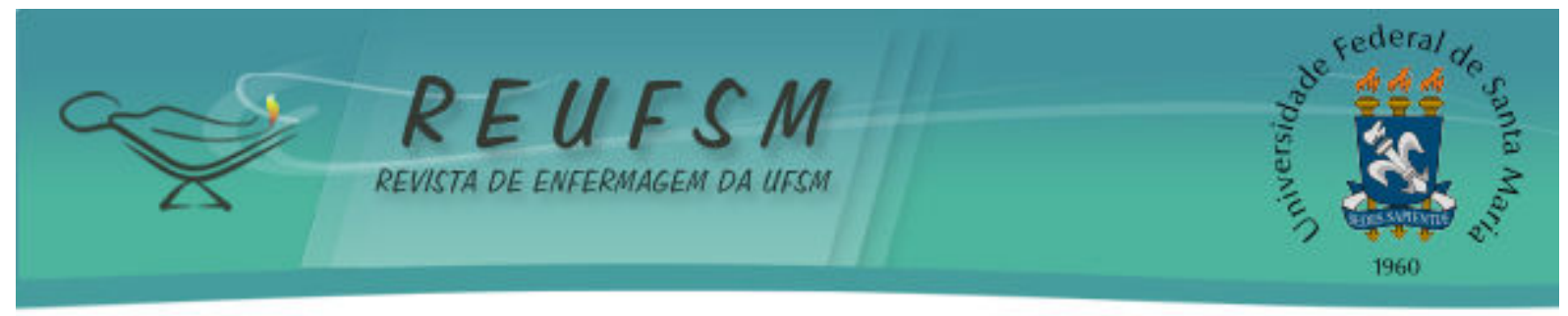

16. Schoeller SD, Leopardi MT, Ramos FS. Cuidado, eixo da vida, desafio para a enfermagem. Rev Enferm UFSM [Internet]. 2011 [cited 2014 Nov 4]; 1(1):88-96. Available from:http://cascavel.ufsm.br/revistas/ojs-2.2.2/index.php/reufsm/article/view/2013/1515.

17. Virani A, Green T, Turin TC. Phantom limb pain: a nursing perspective. Nursing Standard [Internet]. 2014 [cited 2015 Mai 4]; 29(1):44-50. Available from:http://journals.rcni.com/doi/abs/10.7748/ns.29.1.44.e8730.

18. Brasil. Diretrizes para o cuidado das pessoas com doenças crônicas nas redes de atenção à saúde e nas linhas de cuidado prioritárias. MS. Secretaria de Atenção à Saúde. Departamento de Atenção Básica. Brasília. 2013; 30.

19. Vargas MAO, Ferrazzo S, Schoeller SD, Drago LC, Ramos FRS. Rede de atenção à saúde à pessoa amputada. Rev Acta Paulista Enferm. São Paulo. 2014; 27(6): 526-36.

20. Evans CB. Content Analyses of A Priori Qualitative Phantom Limb Pain Descriptions and Emerging Categories in Mid-Southerners with Limb Loss. Rehabilitation Nursing. 2014;39: 207-215. DOI: 10.1002/rnj.84.

21. Kurichi JE, Xie D, Kwong LP, Bates BE, Vogel WB, Stineman MG. Factors associated with late specialized rehabilitation among veterans with lower extremity amputation who underwent immediate postoperative rehabilitation. Am J Phys Med Rehabil [Internet]. 2011 [cited 2014 Jun 18]. 387-398. Avaliable from: http://www.ncbi.nlm.nih.gov/pmc/articles/PMC3116952/.

22. Brasil. Portaria $n^{\circ} 963$, de 27 de maio de 2013. Redefine a Atenção Domiciliar no âmbito do Sistema Único de Saúde. Ministério da Saúde. Departamento de Atenção Básica. Brasília. 2013.

23. Pereira JATP. Papel/competências do enfermeiro de reabilitação em Unidades de Cuidados Intensivos. In: Gomes B, Rocha MC, Martins MM, Gonçalves MN, organizadores. Investigação em enfermagem de reabilitação: um novo conhecimento para guiar a prática de cuidados. Esc Sup Enferm do Porto [Internet]. 2014 [acesso em 2015 jan 18]:64-72. Disponível em: http://www.esenf.pt/fotos/editor2/i_d/publicacoes/978-898-98443-1-5.pdf.

Data de recebimento: 03/06/2015

Data de aceite: 02/03/2016

Autor responsável: Micheli Leal Ferreira

Endereço postal: Servidão Diocício Permino Ferreira, nr 02, Apto 02

Vargem Grande - Florianópolis/SC CEP: 88052620

E-mail: micheli_leal@yahoo.com.br 